\title{
Experiencia piloto para incorporar la ética informática de forma transversal en el Grado de Ingeniería Informática Pilot experience to mainstream computer ethics in the Computer Science Degree
}

\author{
Alicia García-Holgado ${ }^{1}$, Francisco J. García-Peñalvo ${ }^{1}$, Roberto Therón ${ }^{1}$, Andrea Vázquez-Ingelmo ${ }^{1}$, Adriana \\ Gamazo $^{2}$, Carina S. González-González ${ }^{3}$, Rosa María Gil Iranzo ${ }^{4}$, Ismar Frango Silveira ${ }^{5}$, Marc Alier Forment ${ }^{6}$ \\ aliciagh@usal.es, fgarcia@usal.es, theron@usal.es, andreavazquez@usal.es, adrianagamazo@usal.es, cjgonza@ull.edu.es, \\ rgil@diei.udl.cat, ismarfrango@gmail.com, marc.alier@upc.edu
}

${ }^{1}$ Dpto. de Informática y Automática, Grupo de Investigación GRIAL, IUCE

Universidad de Salamanca Salamanca, España

${ }^{4}$ Dpto. de Informática e Ingeniería Industrial

Universitat de Lleida Lleida, España

\author{
${ }^{2}$ Dpto. de Didáctica, Organización y \\ Métodos de Investigación, \\ Grupo de Investigación GRIAL, IUCE \\ Universidad de Salamanca \\ Salamanca, España
}

${ }^{5}$ Faculdade de Computação e Informática

Universidade Presbiteriana Mackenzie Sao Paulo, Brasil
${ }^{3}$ Dpto. de Ingeniería Informática y de Sistemas, Instituto Universitario de

Estudios de las Mujeres

Universidad de la Laguna

San Cristóbal de la Laguna, España

${ }^{6}$ Dpto. Ingeniería de Servicios y Sistemas de Información Grupo de Investigación BCN-SEER Universitat Politecnica de Catalunya Barcelona, España

\begin{abstract}
Resumen- Cuando se realiza cualquier desarrollo software se deben tener en cuenta una serie de implicaciones éticas relacionadas con el impacto que ese desarrollo puede tener en los valores humanos y sociales. En este sentido, de acuerdo con el ACM/IEEE-CS Computer Science Curriculum 2013, los graduados deben ser capaces de reconocer las cuestiones sociales, jurídicas, éticas y culturales inherentes a la disciplina de la informática. El presente trabajo describe una experiencia de innovación docente cuyo objetivo es incorporar los aspectos éticos relacionados con el desarrollo software en el Grado en Ingeniería Informática a través de un SPOC (Small Private Open Course). Para ello, se han realizado un conjunto de charlas online en torno al temario del SPOC, de tal forma que al finalizar el curso, se dispone de los recursos base para crear un conjunto de píldoras de vídeo.
\end{abstract}

Palabras clave: ética informática, ingeniería, educación superior, SPOC

\begin{abstract}
A number of ethical implications related to the impact that such development may have on human and social values must be taken into account when undertaking any software development. In this sense, according to the ACM/IEEE-CS Computer Science Curriculum 2013, graduates must be able to recognise the social, legal, ethical and cultural issues inherent to the discipline of computer science. This work describes a teaching innovation experience whose objective is to incorporate the ethical aspects related to software development in the Degree in Computer Engineering through a SPOC (Small Private Open Course). For this purpose, a set of online talks have been carried out around the SPOC's topics, in order to create a set of video pills at the end of the course.
\end{abstract}

Keywords: computer ethics, engineering, higher education, SPOC

\section{INTRODUCCIÓN}

Las nuevas tecnologías desempeñan un papel fundamental en el mercado laboral actual y futuro en todos los sectores, tanto a nivel económico como industrial. Aunque se utilizan diferentes términos para hablar del futuro próximo, como Industria $4.0 \mathrm{o}$ Sociedad 5.0 (Nakanishi, 2019), todos consideran la transformación de las industrias en favor de las competencias tecnológicas (De Bruyne \& Gerritse, 2018; World Economic Forum, 2016).

A pesar de la automatización de los procesos gracias al desarrollo tecnológico, el factor humano sigue siendo necesario. De acuerdo con Oliveira et al. (2019), en la Industria 4.0, cada vez se necesita más personal, no solo con habilidades y conocimientos relacionados con la tecnología (Pinzone et al., 2017), sino también con competencias transversales tales como la creatividad y la resolución de problemas. El informe de 2018 sobre el futuro del empleo, planteaba que para 2022 el dominio de las nuevas tecnologías representaría solo una parte de las habilidades más demandadas, siendo las competencias "humanas" como la creatividad, la originalidad y la iniciativa, el pensamiento crítico, la persuasión y la negociación las que se mantendrían o aumentarían su valor, al igual que la atención a los detalles, la resiliencia, la flexibilidad y la resolución de problemas complejos (World Economic Forum, 2018).

Estas premisas se ratifican en el último informe publicado (World Economic Forum, 2020) donde destacan las competencias transversales entre las quince competencias más demandadas por los empleadores para 2025, situándose la innovación, el pensamiento crítico o la resolución de problemas 
complejos por encima del uso y diseño de la tecnología. Asimismo, la integridad, la confianza y la atención al detalle son habilidades muy demandadas en un gran número de países.

Estas habilidades toman especial relevancia si hablamos de trabajadores en el ámbito del desarrollo tecnológico ya que se deben tener en cuenta una serie de implicaciones éticas relacionadas con el impacto que ese desarrollo puede tener en los valores humanos y sociales. En 2025, se estima que el tiempo dedicado a las tareas actuales en el trabajo por los humanos y las máquinas será igual (World Economic Forum, 2020).

De acuerdo con el Computer Science Curriculum 2013 elaborado por dos de las principales asociaciones en cuestiones de tecnología e ingeniería (ACM \& IEEE Computer Society, 2013), los graduados en ciencias de la computación deben tener una serie de habilidades personales no solo centradas en las cuestiones tecnológicas, sino también en las cuestiones sociales, jurídicas, éticas y culturales inherentes a la disciplina de la informática.

A pesar de que los aspectos sociales y éticos de la tecnología se han incluido en algunos planes de estudios de informática desde hace más de veinte años, existe una gran variedad entre los cursos de ética informática en cuanto a los contenidos que se imparten (Fiesler et al., 2020; Nasir et al., 2021) y la forma de incorporarlos en el currículo (Casañ et al., 2020). Asimismo, existe una falta de integración de la ética informática en el contexto español. Si se observa la base de datos colaborativa sobre asignaturas o programas de educación superior que trabajan la "ética tecnológica" (Fiesler, 2018), el número de universidad españolas es muy inferior al resto de países.

En el caso de la Universidad de Salamanca, concretamente en el Grado de Ingeniería Informática, no no existe ninguna asignatura que trabaje la ética informática. Si bien existen asignaturas en las que se trabaja cuestiones de diversidad e inclusión, no se aborda ninguna de las materias desde el punto de vista ético.

En este contexto, el presente trabajo describe una prueba piloto que se ha llevado a cabo en el Grado de Ingeniería Informática de la Universidad de Salamanca a través de un proyecto de innovación docente financiado en el curso 2020-21 que ha permitido generar una serie de contenidos relacionados con la ética informática así como recoger la percepción de los participantes para mejorar la propuesta en próximos cursos.

El principal objetivo de la experiencia ha sido introducir el concepto de ética informática así como las diferentes implicaciones que puede tener el desarrollo software en la sociedad.

\section{CONTEXTO}

El objetivo general del proyecto de innovación es incorporar los aspectos éticos relacionados con el desarrollo software en el Grado en Ingeniería Informática a través de un SPOC (Small Private Open Course) cuya elaboración se ha llevado a cabo a lo largo del curso académico 2020-21. En particular, los materiales del SPOC se han elaborado a través de las grabaciones de un conjunto de charlas online en torno al temario del SPOC. De esta forma, durante el desarrollo del proyecto se han elaborado y pilotado los materiales y el enfoque del SPOC, el cual queda disponible para su uso en cursos posteriores por los estudiantes de disciplinas relacionadas con la informática.
Para la consecución del objetivo general planteado se han definido los siguientes objetivos específicos:

- Realizar el diseño instruccional del SPOC.

- Diseñar y grabar charlas online sobre diferentes aspectos éticos en el desarrollo software como parte de las asignaturas Ingeniería de Software I e Interacción Persona-Ordenador.

- Crear e implementar el SPOC en las asignaturas señaladas.

El piloto se ha llevado en el segundo cuatrimestre del curso 2020-21 en dos asignaturas obligatorias del Grado de Ingeniería Informática. Concretamente en el grupo A de la asignatura de Ingeniería de Software I que se imparte en el segundo curso, y en todos los grupos de la asignatura Interacción PersonaOrdenador que se imparte en el tercer curso.

Estas asignaturas se han elegido porque, además de realizarse en el mismo periodo de tiempo y abarcar los dos cursos centrales del Grado, proporcionan un contexto adecuado para integrar cuestiones de ética informática como parte de su temario. Además, en el caso de Ingeniería de Software I, integra desde el curso 2016-17 algunos aspectos éticos relacionados con la inclusión y la diversidad en el proceso de desarrollo (García-Holgado et al., 2020; García-Holgado et al., 2021), aunque no se ha abordado de forma directa la ética informática. Por otro lado, la asignatura de Interacción Persona-Ordenador acerca el desarrollo software a la sociedad a través del Diseño Centrado en el Usuario.

\section{DESCRIPCIÓN}

\section{A. Elaboración del SPOC}

El objetivo principal del SPOC es proporcionar a los estudiantes de ingeniería informática una base de conocimiento sobre ética en el desarrollo software. El temario del SPOC se divide en cuatro grandes bloques: introducción a la ética informática, ética e interacción persona-ordenador, ética y diseño universal, y ética e inteligencia artificial.

Las píldoras de vídeo para cada uno de los bloques se han realizado mediante un conjunto de charlas online. Las charlas, impartidas por expertos nacionales e internaciones en cuestiones éticas en informática, tienen una duración máxima de 40 minutos con una estructura en bloques que permita posteriormente editar las grabaciones y crear las píldoras de vídeo. Cada charla se divide en tres partes: exposición de la persona experta, preguntas y cuestionario sobre la charla.

La primera charla, "Introducción a la ética informática", fue realizada por el Dr. Marc Alier Forment, docente en la Universidad Politécnica de Cataluña (España) con más de 30 años de experiencia docente en ética y sostenibilidad en informática.

La segunda charla, "Ética e Interacción Persona-Ordenador", se centra en la ética informática en cuestiones relacionadas con la interacción persona-ordenador y el diseño centrado en el usuario. La charla fue impartida por la Dra. Carina Soledad González González, catedrática de la Universidad de La Laguna (España). Durante más de 20 años, su investigación se ha centrado en el campo de la Informática aplicada a la Educación y la Interacción Persona-Ordenador, participando en diferentes 
proyectos de investigación y publicando ampliamente sobre estos temas.

Respecto a la tercera charla, "Ética y diseño universal", abordó el diseño ético y universal de la mano del Dr. Ismar Frango, docente en la Universidad Presbiteriana Mackenzie (Brasil) y en la Universidade Cruzeiro do Sul (Brasil). Por último, la cuarta charla, "Ética e Inteligencia Artificial", ha acercado la ética informática a la inteligencia artificial (IA), haciendo especial hincapié en la IA aplicada a la robótica. La charla fue realizada por la Dra. Rosa María Gil Iranzo, experta en ética e inteligencia artificial de la Universidad de Lleida (España).

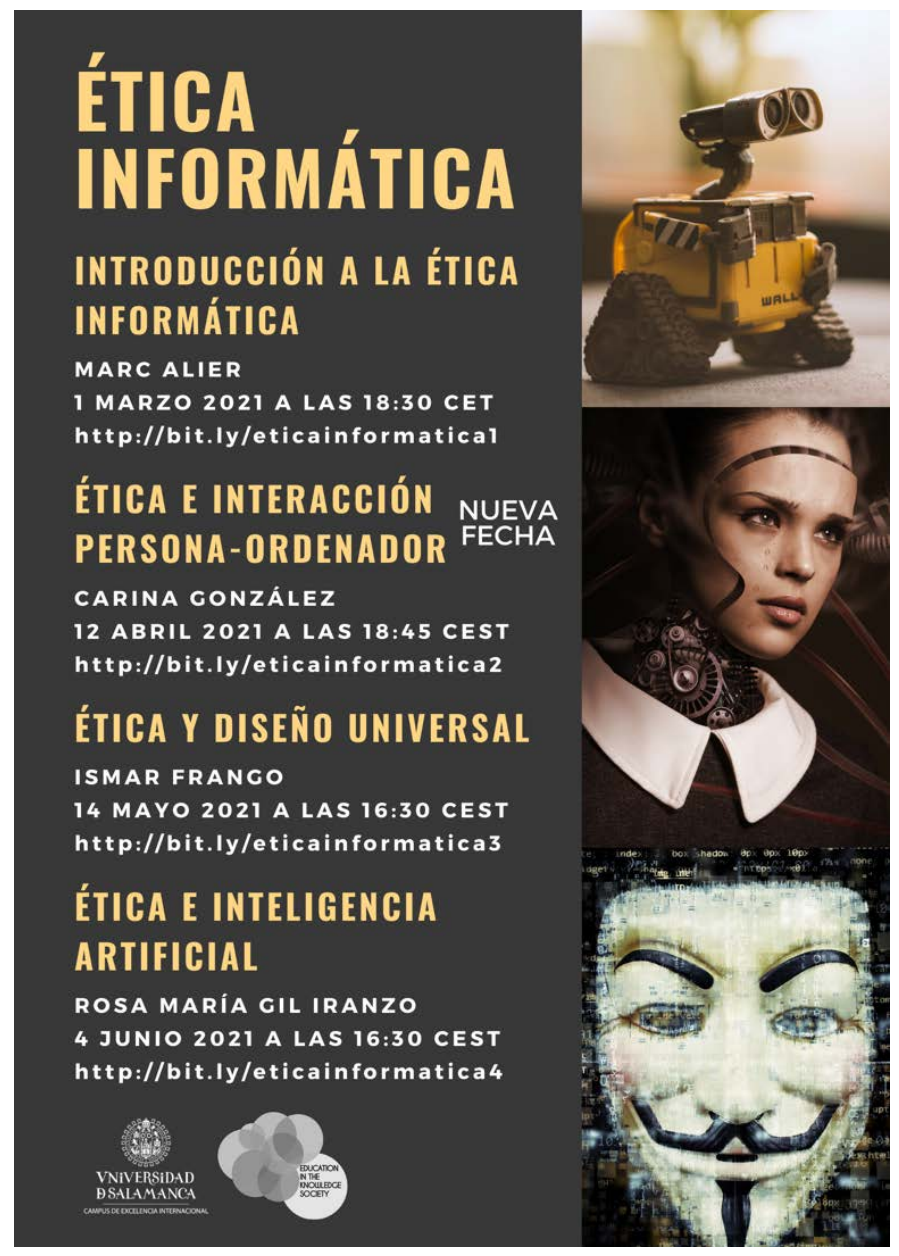

Figura 1. Programación de charlas

Las charlas se programaron a lo largo del segundo cuatrimestre (Figura 1), teniendo en cuenta los periodos de vacaciones y las pruebas de evaluación continua del alumnado. Debido a que las charlas involucran alumnado de dos cursos, se realizaron en horario no lectivo. El orden de las charlas es el mismo orden que mantienen los contenidos en el SPOC, de tal forma que el alumnado que ha participado en las charlas ha adquirido las nociones básicas sobre ética informática.

Aunque los destinatarios de las charlas son los estudiantes de segundo y tercero del Grado en Ingeniería Informática, a través de las dos asignaturas involucradas, Ingeniería de Software I e Interacción Persona-Ordenador, las charlas se realizaron en formato abierto con la colaboración del Programa de Doctorado Formación en la Sociedad del Conocimiento (García-Holgado et al., 2015; García-Peñalvo, 2013, 2014, 2015). La colaboración con el Programa de Doctorado no solo ha permitido que más personas se beneficien de las charlas, sino que también ha permitido avalar la participación en las mismas con un certificado emitido por un Programa Oficial dentro de la Universidad de Salamanca.

Las charlas se han realizado a través de Zoom con la cuenta institucional y se ha habilitado la inscripción para poder gestionar quiénes se interesaban por las charlas y poder darles mayor difusión así como contactarles con recordatorios previos a la charla. Además, para poder facilitar compartir los enlaces a cada charla se ha utilizado un acortador de enlaces que permitía personalizar el enlace, tal como se muestra en la Figura 1.

\section{B. Integración en las asignaturas}

Además del diseño del SPOC y la realización de las charlas, otra de las principales tareas que afectan a los resultados del piloto es la integración de la propuesta en las asignaturas involucradas en el proyecto.

Con objeto de integrar la elaboración del SPOC como parte de las asignaturas, se han incorporado los aspectos éticos en los trabajos finales que se realizan en cada una de las asignaturas. Estos trabajos se desarrollan a lo largo de todo el cuatrimestre por lo que tienen un peso muy importante en el proceso de aprendizaje. En particular, se ha añadido un indicador de ética en las rúbricas de evaluación de ambos trabajos. Cómo alcanzar la máximo puntuación en dicho indicador ha formado parte del proceso de aprendizaje, de tal forma que el alumnado ha tenido que ir conectando los diferentes elementos que se trabajan en cada asignatura para poder entenderlo y aplicarlo, siendo especialmente útil asistir a las charlas organizadas en horario no lectivo.

Además, para valorar la participación del alumnado, se estableció como requisito asistir al menos a dos charlas y responder un cuestionario final sobre percepción de la ética informática para obtener el certificado de superación del SPOC.

Si bien el alumnado sabía que la ética era un aspecto a valorar en la rúbrica de sus trabajos finales en ambas asignaturas, la participación en las charlas ha sido voluntaria. Por este motivo se estableció una estrategia de difusión e integración del SPOC en los espacios online de cada asignatura.

En primer lugar, se elaboró un vídeo de presentación de las charlas adaptado a cada asignatura de tal forma que, además de presentar las charlas que se iban a realizar, se indicaba cómo esto iba a impactar en el trabajo final de cada asignatura.

Por otro lado, se realizó cartelería para acompañar la información sobre las charlas dentro del espacio virtual del curso (Figura 1). En la asignatura de Ingeniería de Software I se creó una sección específica en Studium, el campus virtual de la Universidad de Salamanca basado en Moodle, con objeto de proporcionar toda la información sobre las charlas así como dar acceso a las grabaciones de cada una de ellas (Figura 2). En cuanto a Interacción Persona-Ordenador, la mayor parte de la interacción con el alumnado se realiza en un equipo en Microsoft Teams, de tal forma que toda la información se les hizo llegar a través del canal general (Figura 3).

En tercer lugar, se utilizó el portal del Programa de Doctorado Formación en la Sociedad del Conocimiento para dar visibilidad a las charlas, de tal forma que también se abrió la participación a estudiantes de doctorado interesados en 
conocer qué es la ética informática (https://knowledgesociety.usal.es/seminars/ética-informática).

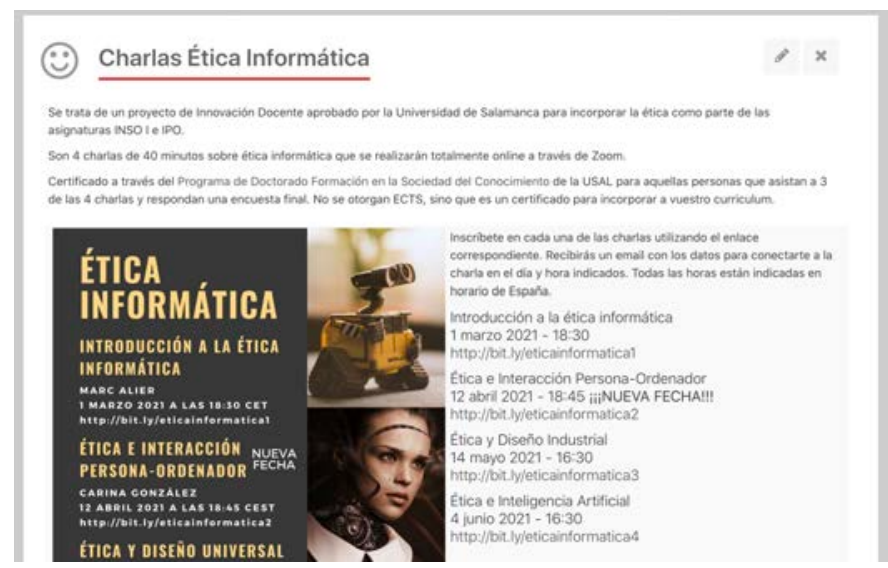

Figura 2. Espacio del proyecto en el campus virtual de Ingeniería de Software I

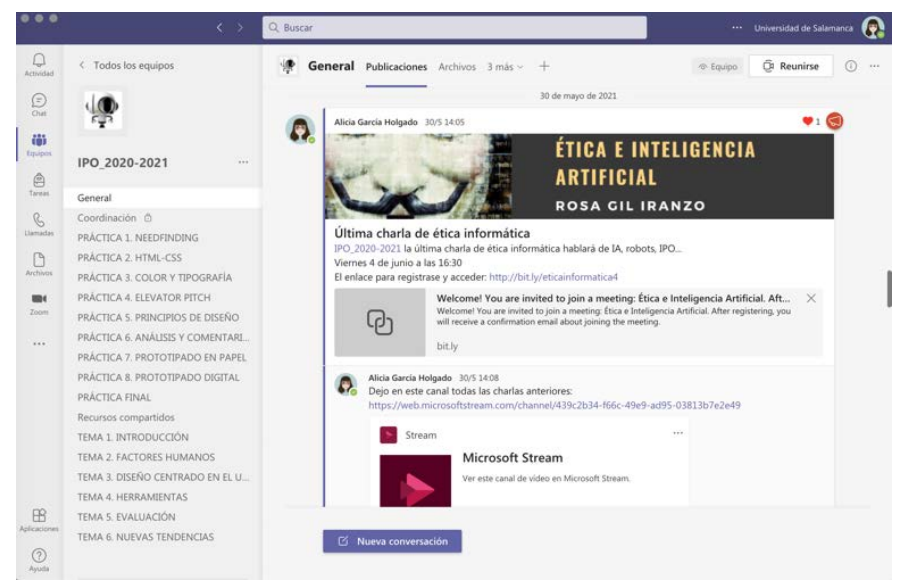

Figura 3. Espacio del proyecto en el espacio virtual de Interacción Persona-Ordenador en Microsoft Teams

\section{Evaluación de los resultados}

La evaluación se ha divido en dos partes. En primer lugar, una evaluación para medir la satisfacción de los estudiantes respecto a las charlas y la integración de los aspectos éticos en las asignaturas. Para ello, se ha elaborado un instrumento a medida con siete ítems de tipo Likert de cinco puntos donde 1 es "muy en desacuerdo" y 5 es "totalmente de acuerdo":

Q1. En general considero que la charla ha sido satisfactoria.

Q2. Recomendaría la charla a personas con mis mismos intereses.

Q3. Asistiría a más charlas del/la mismo/a ponente.

Q4. Las explicaciones del/la ponente me han resultado claras y comprensibles.

Q5. La duración de la charla ha sido adecuada.

Q6. Tengo interés en participar en actividades de las mismas características sobre otros temas.

Q7. El contenido de la charla ha sido interesante.

El cuestionario se satisfacción se ha lanzado a través del sistema de votaciones de Zoom al finalizar cada una de las charlas, de tal forma que la mayoría de los participantes han respondido el cuestionario. La recogida de datos se ha realizado de forma anónima.

Por otro lado, se ha completado la evaluación con un cuestionario final para medir la actitud de los estudiantes frente a una serie de cuestiones éticas. En este caso, tras un análisis de diferentes instrumentos relacionados con la ética informática, se eligió la última versión disponible de "2009 ETHICOMP® Survey of Ethical Attitudes" (Prior et al., 2010) al adaptarse mejor a las características de la población objetivo. En total se compone de 27 ítems y un conjunto muy amplio de preguntas demográficas, sobre todo enfocadas al ámbito profesional, a pesar de que el instrumento también se puede utilizar en contexto educativo. En particular, se han tomado los veinticuatro ítems Likert de cinco puntos (1-24), descartando aquellos que van dirigidos a profesionales del sector. Además, los ítems se han traducido al español. La población objetivo han sido aquellas personas que han participado en dos o más charlas.

\section{Resultados}

\section{A. Descripción de la muestra}

En primer lugar, se presenta un conjunto de indicadores que describen la población piloto y la muestra. Respecto a la población, el número de estudiantes que cursan Ingeniería de Software I en el Grupo A es de 80 matriculados y en Interacción Persona-Ordenador de 144 matriculados. En el programa de doctorado hay 179 matriculados.

Respecto a la muestra, el número de estudiantes únicos que han asistido a las charlas es de 101, siendo la distribución por charla como se muestra en la Tabla 1. De este total, únicamente 24 participantes no pertenecen al Grado de Ingeniería Informática.

Tabla 1. Participantes en las charlas online

\begin{tabular}{lcc}
\hline \multicolumn{1}{c}{ Charla } & Inscritos & Asistentes \\
\hline Introducción a la ética informática & 103 & 73 \\
\hline Ética e Interacción Persona-Ordenador & 97 & 51 \\
\hline Ética y diseño universal & 79 & 44 \\
\hline Ética e Inteligencia Artificial & 70 & 38 \\
\hline
\end{tabular}

El número de estudiantes va disminuyendo a medida que finaliza el curso. Esta situación no solo puede deberse a falta de interés por parte del alumnado, sino que también coincide con el periodo de mayor actividad en cuanto a pruebas de evaluación continua y entregas de prácticas durante el mes de mayo. Varios estudiantes que habían participado en las charlas previas solicitaron poder acceder a los vídeos posteriormente por incompatibilidad horaria.

En cuanto al porcentaje de estudiantes que finalmente han asistido a dos o más charlas, representan un 58,42\% del total de participantes. En concreto, 59 estudiantes de los cuáles 10 provienen de fuera del Grado.

Tabla 2. Participantes en el cuestionario de satisfacción

\begin{tabular}{lcc}
\hline \multicolumn{1}{c}{ Charla } & Respuestas & Porcentaje \\
\hline Introducción a la ética informática & 72 & $98,63 \%$ \\
\hline Ética e Interacción Persona-Ordenador & 50 & $98,04 \%$ \\
\hline Ética y diseño universal & 39 & $88,64 \%$ \\
\hline Ética e Inteligencia Artificial & 24 & $63,16 \%$ \\
\hline
\end{tabular}


Finalmente, respecto a la satisfacción de los participantes la muestra recogida en cada una de las charlas se puede ver en la Tabla 2. Se debe aclarar que la baja participación en el cuestionario de la última charla se debe a un problema técnico que finalizó la disponibilidad de la votación en Zoom antes de tiempo.

\section{B. Satisfacción de los participantes}

Se han comparado los resultados obtenidos en cada una de las charlas utilizando la prueba de Kruskal Wallis debido a que se trata de muestras independientes que no siguen una distribución normal. El análisis muestra diferencias significativas únicamente en el primer ítem Q1 (En general considero que la charla ha sido satisfactoria) con $p=, 014$ para un nivel de significancia de ,05. En la Figura 4 se muestra la distribución de las respuestas para cada una de las charlas.

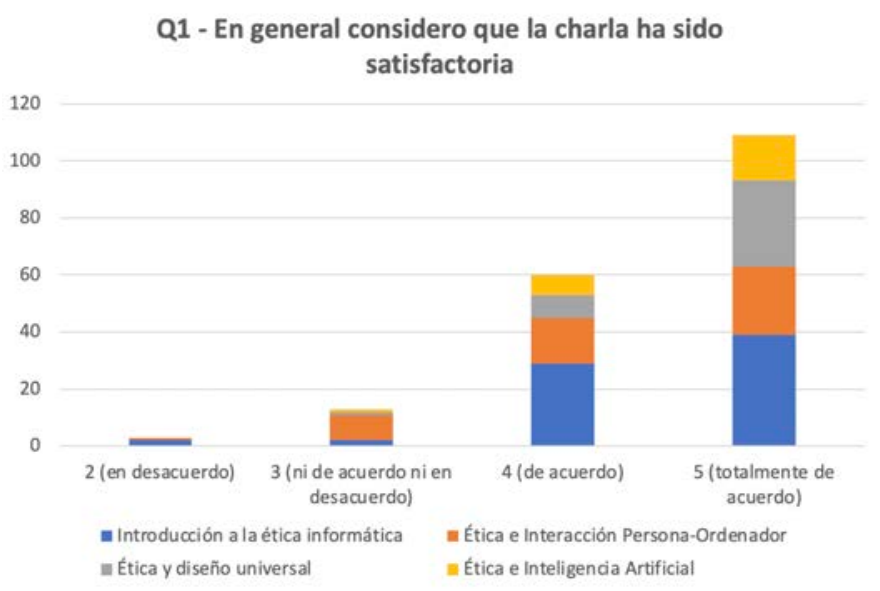

Figura 4. Resultados de la pregunta Q1 $(\mathrm{N}=185)$

En términos generales, la satisfacción de los participantes es alta en todos los ítems del cuestionario para todas las charlas, encontrándose la media de respuestas entre 4 y 5 (de acuerdo y totalmente de acuerdo). Analizando la frecuencia de las respuestas para cada una de las preguntas, el porcentaje de total de respuestas con valor 5 (totalmente de acuerdo) para $\mathrm{N}=185$ oscila entre $58,9 \%$ y $66,5 \%$.

Finalmente, destacan los resultados de la pregunta Q6 (Tengo interés en participar en actividades de las mismas características sobre otros temas), donde el 94\% de los participantes para $\mathrm{N}=185$ está de acuerdo con la afirmación y solo el $1,1 \%$ está en desacuerdo.

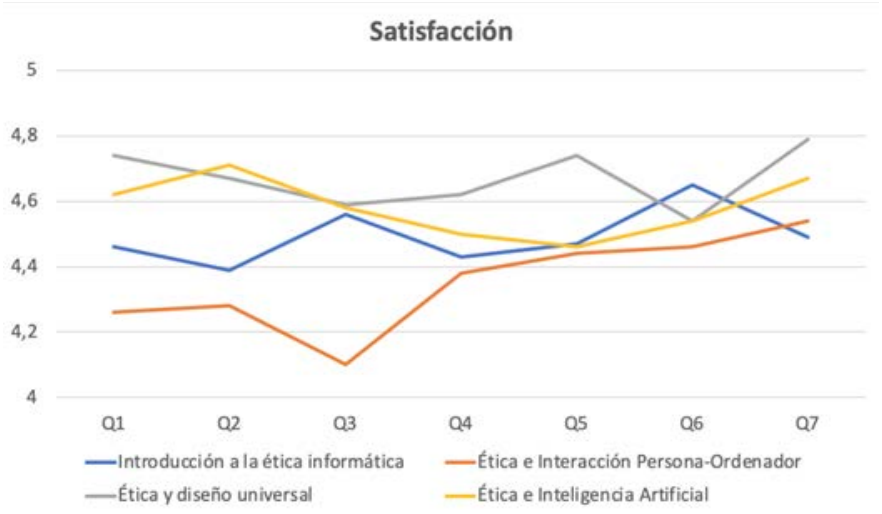

Figura 5. Medias de las preguntas de satisfacción para cada charla

\section{CONCLUSIONES}

A lo largo del curso académico 2020-21 se ha llevado a cabo una experiencia piloto centrada en desarrollar un SPOC sobre ética informática de forma incremental. El contenido del SPOC se ha elaborado mediante charlas online impartidas por expertos nacionales e internacionales.

El carácter innovador de esta propuesta reside, por un lado, en la incorporación de la ética en informática como parte de una titulación oficial, donde no existe ninguna asignatura que trate estos conceptos. Por otro lado, se ha utilizado un enfoque online que permite adaptarse a los diferentes escenarios generados por la actual crisis sanitaria (Fardoun et al., 2020; García-Peñalvo et al., 2020; García-Peñalvo et al., 2021), a la par que generar una base de contenidos en vídeo que se integran en el SPOC. Finalmente, el SPOC quedará disponible para su utilización en cursos posteriores, principalmente para estudiantes del Grado en Ingeniería Informática y el Grado en Ingeniería Informática en Sistemas de Información, pudiendo ser considerado una buena práctica en educación superior.

Respecto a los resultados obtenidos, cabe destacar la alta satisfacción de los participantes, lo que permite valorar positivamente el contenido del SPOC como una herramienta para introducir la ética informática. Todas las charlas han tenido valores altos de satisfacción tanto por el contenido como por los ponentes. Además, los resultados muestran un alto grado de interés por actividades similares con tan solo un $1,1 \%$ que no están satisfechos.

En cuanto a las limitaciones del proyecto de innovación, es importante destacar la dificultad de organizar el contenido de las charlas para luego elaborar píldoras de vídeo más pequeñas, lo que ha llevado a crear píldoras de vídeo de mayor duración que la esperada inicialmente. Asimismo, es necesario probar el resultado final del SPOC con estudiantes de cursos próximos para validar los resultados preliminares obtenidos.

\section{AgRADECIMIENTOS}

Este trabajo es parte del proyecto de innovación docente "Diseño y creación de un SPOC sobre consideraciones éticas en el desarrollo software para los estudiantes del Grado en Ingeniería Informática" (ID2020/002) financiado por la Universidad de Salamanca (España) en el curso 2020-21; y parcialmente apoyado por el Ministerio de Ciencia, Innovación y Universidades de España a través de una beca FPU (FPU017/01252).

\section{REFERENCIAS}

ACM, \& IEEE Computer Society. (2013). Computer Science Curricula 2013. doi:10.1145/2534860

Casañ, M. J., Alier, M., \& Llorens, A. (2020). Teaching Ethics and Sustainability to Informatics Engineering Students, An Almost 30 Years' Experience. Sustainability, 12(14), 5499. doi:10.3390/su12145499

De Bruyne, E., \& Gerritse, D. (2018). Exploring the future workplace: results of the futures forum study. Journal of Corporate Real Estate, 20(3), 196-213. doi:10.1108/JCRE-09-2017-0030

Fardoun, H., González-González, C. S., Collazos, C. A., \& Yousef, M. (2020). Exploratory Study in Iberoamerica on the Teaching-Learning Process and Assess- 
ment Proposal in the Pandemic Times. Education in the Knowledge Society, 21. doi:10.14201/eks.23437

Fiesler, C. (2018). Tech Ethics Curricula: A Collection of Syllabi. Medium. Recuperado el 25 de agosto de 2021 de https://medium.com/@cfiesler/tech-ethicscurricula-a-collection-ofsyllabi-3eedfb76be18

Fiesler, C., Garrett, N., \& Beard, N. (2020). What Do We Teach When We Teach Tech Ethics? A Syllabi Analysis Proceedings of the 51st ACM Technical Symposium on Computer Science Education, Portland, OR, USA.

García-Holgado, A., García-Peñalvo, F. J., \& RodríguezConde, M. J. (2015). Definition of a Technological Ecosystem for Scientific Knowledge Management in a PhD Programme. En G. R. Alves \& M. C. Felgueiras (Eds.), Proceedings of the Third International Conference on Technological Ecosystems for Enhancing Multiculturality (TEEM'15) (Porto, Portugal, October 7-9, 2015) (pp. 695-700). ACM. doi:10.1145/2808580.2808686

García-Holgado, A., Vázquez-Ingelmo, A., García-Peñalvo, F. J., \& González-González, C. S. (2020). Perspectiva de género y fomento de la diversidad en la docencia de Ingeniería del Software. En Actas de las Jornadas de la Enseñanza Universitaria de la Informática (JENUI) (Vol. 5, pp. 269-276). AENUI, la Asociación de Enseñantes Universitarios de la Informática.

García-Holgado, A., Vázquez-Ingelmo, A., García-Peñalvo, F. J., \& Rodríguez-Conde, M. J. (2021). Improvement of learning outcomes in software engineering: active methodologies supported through the virtual campus. IEEE Revista Iberoamericana de Tecnologias del Aprendizaje, 16(2), 143-153. doi:10.1109/RITA.2021.3089926

García-Peñalvo, F. J. (2013). Aportaciones de la Ingeniería en una Perspectiva Multicultural de la Sociedad del Conocimiento. IEEE VAEP-RITA, 1(4), 201-202.

García-Peñalvo, F. J. (2014). Formación en la sociedad del conocimiento, un programa de doctorado con una perspectiva interdisciplinar. Revista Teoría de la Educación: Educación y Cultura en la Sociedad de la Información, 15(1), 4-9.

García-Peñalvo, F. J. (2015). Engineering contributions to a Knowledge Society multicultural perspective. IEEE Revista Iberoamericana de Tecnologías del Aprendizaje (IEEE RITA), 10(1), 17-18. doi:10.1109/RITA.2015.2391371

García-Peñalvo, F. J., Corell, A., Abella-García, V., \& Grandede-Prado, M. (2020). Online Assessment in Higher Education in the Time of COVID-19. Education in the Knowledge Society, 21. doi:10.14201/eks.23086

García-Peñalvo, F. J., Corell, A., Rivero-Ortega, R., RodríguezConde, M. J., \& Rodríguez-García, N. (2021). Impact of the COVID-19 on Higher Education: An Experience-Based Approach. En F. J. García-Peñalvo (Ed.), Information Technology Trends for a Global and Interdisciplinary Research Community (pp. 1-18). IGI Global.

Nakanishi, H. (2019). Modern society has reached its limits. Society 5.0 will liberate us. World Economic Forum Annual Meeting. https://www.weforum.org/agenda/2019/01/modernsociety-has-reached-its-limits-society-5-0-willliberate-us/

Nasir, O., Muntaha, S., Javed, R. T., \& Qadir, J. (2021, 21-23 April 2021). Work in Progress: Pedagogy of Engineering Ethics: A Bibliometric and Curricular Analysis. 2021 IEEE Global Engineering Education Conference (EDUCON),

Oliveira, M., Arica, E., Pinzone, M., Fantini, P., \& Taisch, M. (2019). Human-Centered Manufacturing Challenges Affecting European Industry 4.0 Enabling Technologies. En C. Stephanidis (Ed.), HCI International 2019 - Late Breaking Papers. HCII 2019. Lecture Notes in Computer Science (Vol. 11786). Springer.

Pinzone, M., Fantini, P., Perini, S., Garavaglia, S., Taisch, M., \& Miragliotta, G. (2017). Jobs and Skills in Industry 4.0: An Exploratory Research. En H. Lödding, R. Riedel, K. Thoben, G. von Cieminski, \& D. Kiritsis (Eds.), Advances in Production Management Systems. The Path to Intelligent, Collaborative and Sustainable Manufacturing. APMS 2017. IFIP Advances in Information and Communication Technology (Vol. 513, pp. 282-288). Springer. doi:10.1007/978-3-31966923-6 33

Prior, M., Fairweather, N. B., Rogerson, S., \& West, D. (2010). IS IT Ethical? 2010 ETHICOMP Survey of Professional Practice.

World Economic Forum. (2016). The future of jobs: Employment, skills and workforce strategy for the fourth industrial revolution. http://www3.weforum.org/docs/WEF_Future_of_Job s.pdf

World Economic Forum. (2018). The future of jobs Report 2018: Centre for the New Economy and Society. World Economic Forum. https://www.weforum.org/reports/the-future-of-jobsreport-2018

World Economic Forum. (2020). The future of jobs Report 2020. World Economic Forum. https://www.weforum.org/reports/the-future-of-jobsreport-2020 